\title{
Disorder induced roughening transition of many elastic lines in a periodic potential
}

\author{
T. Knetter ${ }^{1}$, G. Schröder ${ }^{1}$, M. J. Alava ${ }^{2}$, and H. Rieger ${ }^{3}$ \\ ${ }^{1}$ Institut für Theoretische Physik, Universität zu Köln, 50937 Köln, Germany \\ ${ }^{2}$ Helsinki University of Technology, Laboratory of Physics, P.O.Box 1100, 02015 HUT, Finland \\ 3 Theoretische Physik, Universität des Saarlandes, 66041 Saarbrücken, Germany
}

(November 20, 2018)

\begin{abstract}
The competing effect of a periodic pinning potential and random point disorder is studied for arrays of elastic lines or directed polymers. The groundstates are investigated by exact combinatorial optimization. In both two and three dimensions a phase diagram is found with two or three distinct phases: a strictly flat phase if the disorder is bounded and weak, a weakly fluctuating phase for intermediate valleys depths, where the lines roughen individually on a scale smaller than the line-line distance, and a rough phase for strong disorder, where the roughness follows the scaling with pure point disorder. The line wandering in the transverse direction leads in the three-dimensional, rough phase to an entangled state with a complicated topology.
\end{abstract}

Ensembles of elastic lines form one of the most interesting examples of the interplay of disorder and an ordering tendency. Much of the attention is motivated by the experimental connections to dirty type-II superconductors, in which flux lines (FL) interact with point or columnar defects [1 3 ]. This interaction gives often rise to beneficial, technologically important effects since the impurities pin lines, individually or collectively and creating them in a controlled fashion has been demonstrated by various techniques. The theory of arrays of FLs with a wide variety of possible disorder backgrounds has received lots of interest [4]. It is worth noting that the physics of an individual FL has connections to paradigmatic questions in non-equilibrium statistical mechanics [5.6] through mappings to the Kardar-Parisi-Zhang equation of kinetic roughening in surfaces, and to the Burgers' equation of vortex-free turbulence [7].

Here we analyze with exact numerical tools the roughening or disordering of arrays of elastic lines at zero temperature when point disorder competes with a periodic potential that tries to order the array into a regular structure. The FL analogy is an Abrikosov lattice in the presence of point disorder. For both two- and three-dimensional (2D and 3D) arrays of lines we find a transitions in terms of the ratio $q=\langle\epsilon\rangle / \Delta$, where $\Delta$ measures the depth of the potential valleys, and $\langle\epsilon\rangle$ is the average strength of the point disorder. The picture we gain from our study is in contrast to recent renormalization group studies of a model for elastic periodic media with point disorder in the continuum limit [10,11. Based on these one would expect for an arbitrary disorder strength $q$ in two dimensions asymptotically (in system size) a rough state with diverging FL displacements and line-to-line displacement correlations, whereas we find this to be true only above $q_{c_{2}}$. In other words, the lattice model for a flux line array with a periodic potential shows a roughening transition which should, at least in $2 \mathrm{~d}$, be absent according to analytical theories for an apparently related continuum model. This is also surprising since for the same system without periodic potential good agreement between analytical [12] and numerical 13] results has been achieved. After presenting the numerics we will discuss the apparent contradiction with analytical results.

We are interested in an assembly of $N$ elastic lines described by the Hamiltonian

$$
\begin{aligned}
& \mathcal{H}=\sum_{i=1}^{N} \int_{0}^{H} d z\left\{\frac{\gamma}{2}\left[\frac{d \mathbf{r}_{i}}{d z}\right]^{2}+\sum_{j(\neq i)} V_{\mathrm{int}}\left[\mathbf{r}_{i}(z)-\mathbf{r}_{j}(z)\right]\right. \\
&\left.+V_{r}\left[\mathbf{r}_{i}(z), z\right]+V_{p}\left[\mathbf{r}_{i}(z)\right]\right\} .
\end{aligned}
$$

$\mathbf{r}_{i}(z) \in \mathcal{R}^{d-1}$ (with $d=2$ and $d=3$ studied here) is a displament vector, while $z$ is the longitudinal coordinate in a system of height $H ; V_{r}[\mathbf{r}, z]$ describes the point disorder, which we take to be delta-correlated with variance $\epsilon ; V_{\text {int }}\left[\mathbf{r}-\mathbf{r}^{\prime}\right]$ is a short-range repulsive interaction between the lines (e.g. hard-core) and $V_{p}[\mathbf{r}]$ a periodic potential with period $a$ in all transverse space directions. We assume its minima to be well localized, i.e. they have a width that is small against the interaction range of the lines. This allows only single occupancy of the potential valleys and we concentrate on the case in which the line density $\left(\rho=L^{d-1} / N\right)$ is such that each potential valley is occupied by exactly one elastic line, i.e. $\rho=1 / a^{d-1}$. A similar model has been studied in $d=1+1$ in 14. for the competition between point disorder and random columnar defects, i.e. a potential $V_{p}$ that is not periodic but random.

We study a lattice version of the continuum model (1) that lives on the bonds of a square lattice [15]. The structure of the potential is depicted in Figure 1. We have valleys of effective depth $-\Delta$, and bulk disorder from a probability distribution $P\left(\epsilon_{i j}\right)$ for the bonds of the lattice model. $P$ is taken to be either a flat, bounded distribution so that $\langle\epsilon\rangle=\epsilon_{\max } / 2$, or an unbounded exponential distribution with $\langle\epsilon\rangle=\epsilon$, the decay constant of the distribution. Each bond can only be occupied by a segment of a single line (hard core interactions) and the occupation of each bond $(i j)$ costs a particular amount of 


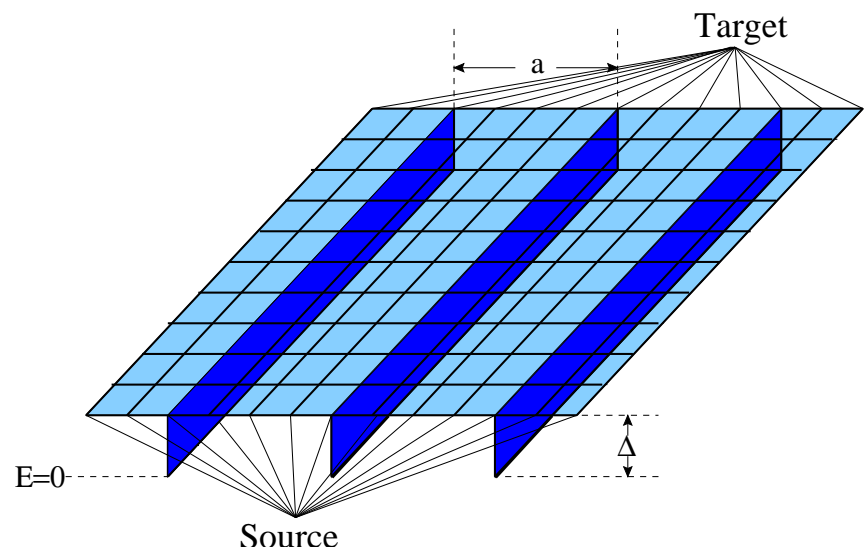

FIG. 1. Periodic potential in $2 D$. The depth of the valleys is denoted by $\Delta$ and the nearest neighbour distance by a. Additional point disorder $\epsilon$ accomplishes the energy landscape. The FL can only enter and leave the system via the energetically neutral arcs connecting the source respectively the sink with the potential valleys.

energy $e_{i j}=\epsilon_{i j}+\Delta_{i j}$ where the $\Delta_{i j}$ 's are zero inside the potential wells and constant elsewhere. This reproduces also the elastic energy, since all bonds cost some positive energy. The lattice Hamiltonian then reads

$$
H(\mathbf{x})=\sum_{(i j)} e_{i j} \cdot x_{i j}
$$

where $\sum_{(i j)}$ is a sum over all bonds $(i j)$ joining site $i$ and $j$ of a $d$-dimensional, e.g. rectangular $\left(L^{d-1} \times H\right)$ lattice, with open boundary conditions (b.c.) in all space directions. The FL enter the system via the plane $z=0$ and leave it via the plane $z=H$, see Fig. 11. The FL configuration is defined by the set of variables $x_{i j}$ that can take on the values $x_{i j}=1$ (if a line is passing bond $(i j)$ and $\left.x_{i j}=0\right)$. For the configuration to form lines on each site of the lattice the configuration $\mathbf{x}$ has to be divergence free (i.e. $\nabla \cdot \mathbf{x}=0$, where $\nabla \cdot$ denotes the lattice divergence). All top-sites are attached to an extra site, via energetically neutral $\operatorname{arcs}, e=0$ : the source with divergence $\nabla \cdot \mathbf{x}=+N$, where $N$ is the number of FLs, and all bottom sites to the target $t$ with $\nabla \cdot \mathbf{x}=-N$. Finding the minimum energy configuration of $N$ lines now becomes equivalent to a minimum cost flow problem with the energy function (2) and the mass balance constraints expressed in the site divergences. This task can be solved exactly in polynomial time (complexity $\mathcal{O}\left(N \cdot L^{d-1} H\right)$ by applying the successive-shortest-path algorithm which is described in detail in 15,17 .

Figure 2 demonstrates with a series of snapshots the geometry involved in the calculations, and the typical behavior with increasing $g$ in $2 d$ and $3 d$. In both cases the lines are pinned to the energetically favorable valleys for small $q$, and finally for large $q$ a cross-over to a rough state takes place. In $3 d$ one can observe that the lines wander almost freely in such conditions.

We discriminate between the different regions in the
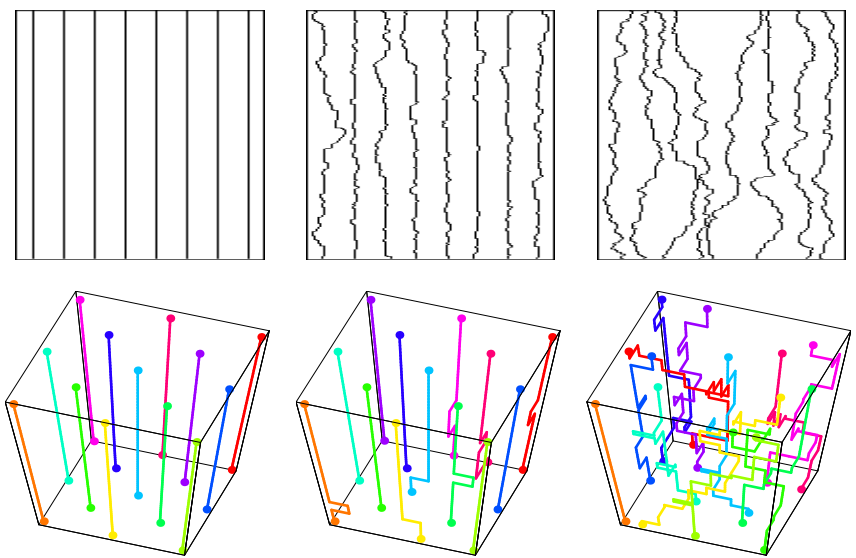

FIG. 2. Optimal ground state configurations in $2 D$ (top) and $3 D$ (bottom) for different point disorder strengths $q$, increasing from left to right. In the flat phase (left) the FL are trapped completely inside the potential valleys.

phase diagram by looking at the behavior of the average transverse fluctuation or roughness $w$ of the lines:

$$
w(L, H)=\left[\frac{1}{N} \sum_{i=1}^{N} \frac{1}{H} \int_{0}^{H} d z\left(\mathbf{r}_{i}(z)-\overline{\mathbf{r}}_{i}\right)^{2}\right]_{\mathrm{av}},
$$

where $\overline{\mathbf{r}}_{i}=H^{-1} \int_{0}^{H} d z \mathbf{r}_{i}(z)$ and $[\ldots]_{\text {av }}$ denotes the disdorder average. By studying very large longitudinal system sizes $H \geq 10^{4}$ we are able to extract the saturation roughness $w(L)=\lim _{H \rightarrow \infty} w(L, H)$ for a finite system of transverse size $L$. Note that we have chosen open b.c.: the transverse fluctuations cannot get larger than the system size. Other quatities of interest are the size $l_{\|}$ of the longitudinal excursions (the average distance between the locations at which a line leaves a valley and returns to it); and the total number of potential valleys $P V$ that a line visits between its entry and terminal point in the limit $H \rightarrow \infty$.

In Fig. 3 we show our data for the roughness $w$ and $l_{\|}$ as a function of $1 / q$ in $2 \mathrm{~d}$, in Fig. $4 w$ and $P V$ as a function of $q$ in $3 \mathrm{~d}$; both for bounded disorder. In Fig. 占 we show our data for $w$ for unbounded disorder. The picture that emerges is the following. In the flat region we have $w(L)=0, l_{\|}=0$ and $P V=1$, i.e. the lines lie completely in the potential valleys. This region $q<q_{c 1}$ exist only for bounded disorder. For the uniform distribution no energetically favourable transverse fluctuation can exist as long as $q<\Delta$. That $q_{c 1}>1$ follows from the fact that we are at full occupancy, $N=N_{V}$ where $N_{V}$ is the number of valleys, for $q \leq q_{c_{1}} \sim 2$ the groundstate consists always of $N$ straight lines regardless of dimension. For unbounded disorder this flat region does not exist, since the probability for a sequence of high-energy bonds in the valleys that pushes the lines out of it is always positive. In the weakly fluctuating region for $q_{c_{1}} \leq q \leq q_{c_{2}}$ the lines roughen locally. Here one has $w>0$ and $l_{\|}>0$, independent of the systems size $L$, and $P V=1$. The transverse fluctuations of flux lines are bounded by the 


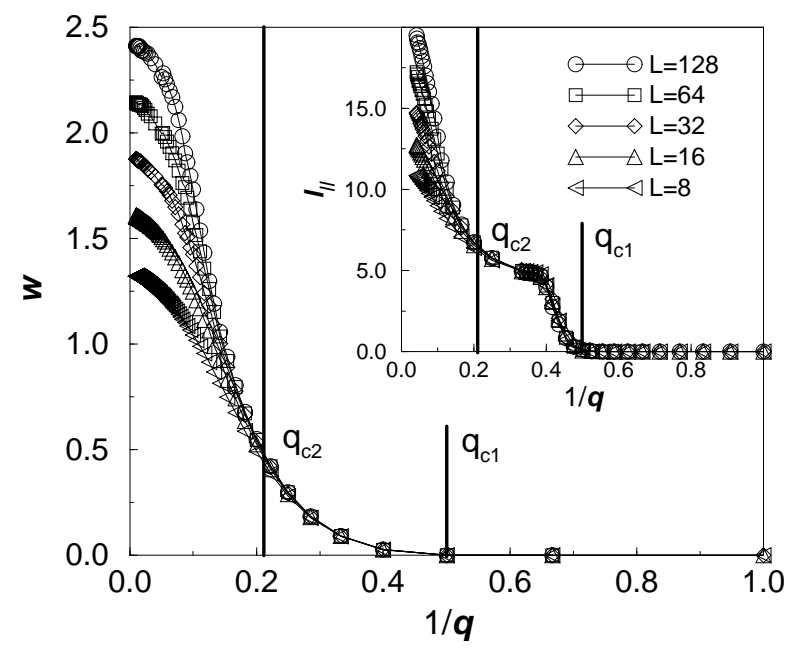

FIG. 3. Roughness $w$ in $2 D$ as a function of disorder strength $q$ for bounded disorder. $q_{c 1}$ and $q_{c 2}$ are shown. In the flat phase $w=0$, whereas $w>0$ for $q>q_{c 1}$. No transversal system size dependence is observered in the weakly fluktuating phase. The inset shows $l_{\|}$. Each data point is averaged over $n=20(L=128)$ up to $n=600(L=8)$ disorder configurations, $a=4$.

average line distance or valley separation $a$. The central feature is that lines fluctuate individually, so that a columnar defect competes with point disorder. Both in $2 \mathrm{D}$ and in $3 \mathrm{D}$ a strong columnar pin strictly localizes the line [8] reducing the line-to-line interaction to zero.

With increasing $q$ the transverse fluctuations increase, and when their lengthscale $l_{\perp}$ becomes comparable to the inter-line distance $a$ the physics changes. When individual lines can jump from one valley to a neighboring valley, i.e. exactly when $P V>1$, a collective rearrangement of the whole line ensemble becomes possible and manifests itself in a system size dependence of the saturation roughness (see Fig. 3 for $2 \mathrm{~d}$ and 14 for $3 \mathrm{~d}$ ). Thus the rough phase is characterized by a dependence of $w$ and $l_{\|}$on the transverse system size and by $P V>1$. In particular the latter criterion facilitates the numerical determination of the location of the roughness transition $q_{c_{2}}$. We find that the critical disorder strength $q_{c_{2}}(a)$ increases monotonically with $a$.

In the limit $q \rightarrow \infty$ the collective behavior of the transverse fluctions of the lines crosses over to that of a line array in the absence of the periodic potential, though the scaling of displacement correlations and the system roughness is slightly decreased compared to the case without the potential because of the lengthscale of the pinned parts of the lines (the remaining tendency to localize in the valleys). For an elastic medium model this would imply that the correlations should depend in a logarithmic fashion on distance. In $2 d$, in particular, it should be $w \sim \ln L[12,13,18$ and our data indicate that this scaling is indeed obtained above $q_{c_{2}}$.

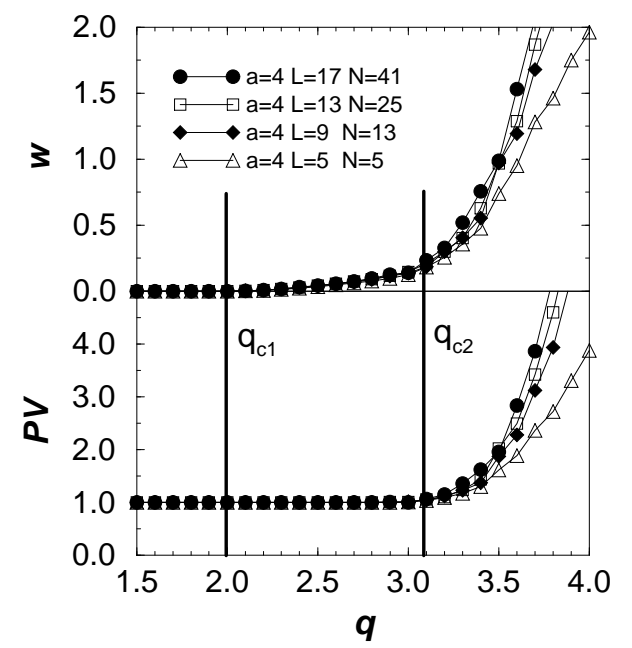

FIG. 4. (Top) The same as in Fig. 月, now in 3d. (Bottom) The FL start to jump from one potential valley to the next at $q_{c 2}$ so that the number of potential valleys each line visits changes from $p v=1$ to $p v>1$ at this threshold (bottom). $n=6(L=128)$ up to $n=100(L=5), a=4$.

A fundamental difference exists between the real-space geometry of two-dimensional and three-dimensional systems. In $3 \mathrm{D}$ and for small line densities, the lines can wander freely since there is no hard-core expulsion in contrast to 2D. This implies that in the thermodynamic limit, the line ensemble becomes entangled. We characterize this by computing the fraction of lines $N_{\text {ent }} / N$ that, when forced to start and end in an arbitrary one of all the potential minima, enter and exit in different valleys. In the limits $H \rightarrow \infty, L \rightarrow \infty N_{\text {ent }}(q) / N$ develops a first-order jump at the roughening transition from zero to unity. $N_{\text {ent }}=N$ implies that all the lines are entangled, though in a finite system the saturation value of $N_{\text {ent }} / N$ is limited by the finite probability that at least one out of $N$ integers ends up at exactly at the same position in a random permutation. This finite size feature can be seen in the data presented in Fig. 6 .

Finally we relate our findings to periodic elastic media with point disorder [11]. These are supposed to represent a coarse grained description of flux line arrays such as (1) and predict the absence of a roughening transition in $2 \mathrm{~d}$ contrary to our results. The reason for this discrepancy is that such models operate in a different limit of many lines between the potential valleys and interactions that renormalize to an elastic constant for the line displacements [18]. Simple scaling considerations show [11] that this model in $d=1+1$ is always rough, essentially due to the fact that the energy for terrace excitations of length scale $L$ in the disorder free case $(\lambda=0)$ has no bulk contributions. On the other hand, in the model (11) we have considered here excitations, e.g. steps of individual lines from one potential valley to the next, do have a bulk contribution, due to the full occupancy of the nar- 


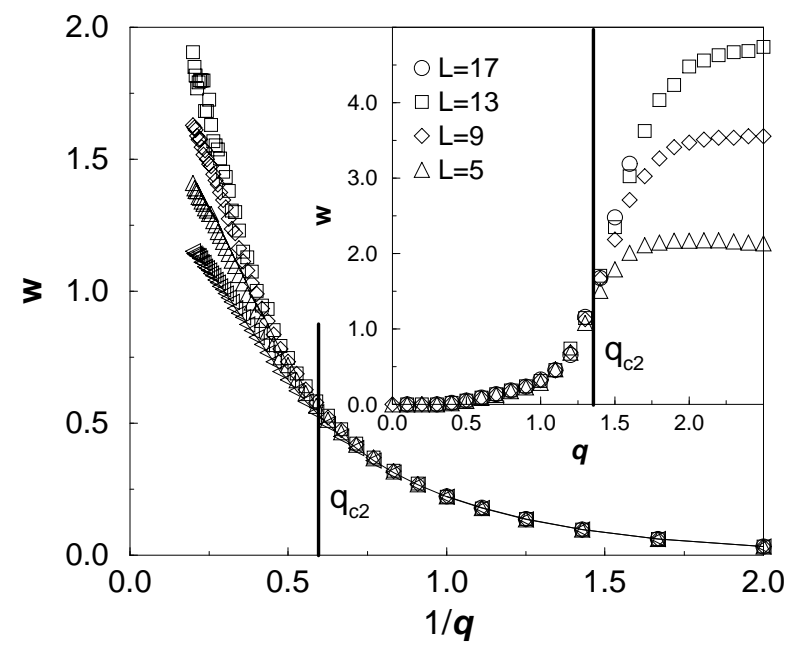

FIG. 5. Same as Fig. A for unbounded disorder. The inset shows $3 D$. Though hardly visible from this data $q_{c 1}$ vanishes. But we still find a finite value of $q_{c 2}$, below which the roughness shows no $L$-dependence both, in $2 D$ and $3 D$. $n=10$ up to $n=200$, both in $2 D$ and $3 D$.

row potential valleys. Therefore we expect a roughening transition for (11) at a non-vanishing disorder strength, as confirmed by our numerical results in $d=2,3$

To conclude, we have analyzed with the aid of exact combinatorial optimization methods ensembles of elastic lines (or directed polymers or FLs) in the presence of a confining periodic potential and competing random point disorder. The main finding is a transition between 'rough' and 'flat regimes, in both two and three dimensions, at a finite potential strength. It arises since even rare fluctuations are not able to induce line-line interactions when the filling factor of the system is at unity. Introducing dislocations (in the form of missing lines) or extra lines is likely to complicate the physics, as in the rough substrate model. In the rough phase the physics is characterized by correlations that increase with system size. In three dimensions we find an entangled phase, in which the lines form a topologically complicated geometric configuration. It needs to be characterized properly, and the effect on the glassy physics should be studied.

This work has been supported by the Academy of Finland and the German Academic Exchange Service (DAAD) within a common exchange project, and separately, by the A. of F.'s Centre of Excellence Programme.

[1] G. Blatter et al., Rev. Mod. Phys. 66, 1125 (1994).

[2] T. Nattermann and S. Scheidl, Adv. Phys. 49, 607 (2000).

[3] Spin Glasses and Random Fields, ed. A. P. Young, (World Scientific, Singapore, 1997).

[4] D. S. Fisher, M. P. A. Fisher, and D. A. Huse, Phys. Rev. B 43, 130 (1991); T. Giamarchi and P. Le Doussal, Phys. Rev. B 52, 1242 (1995).

[5] T. Halpin-Healy and Y.-C. Zhang, Phys. Rep. 254, 215

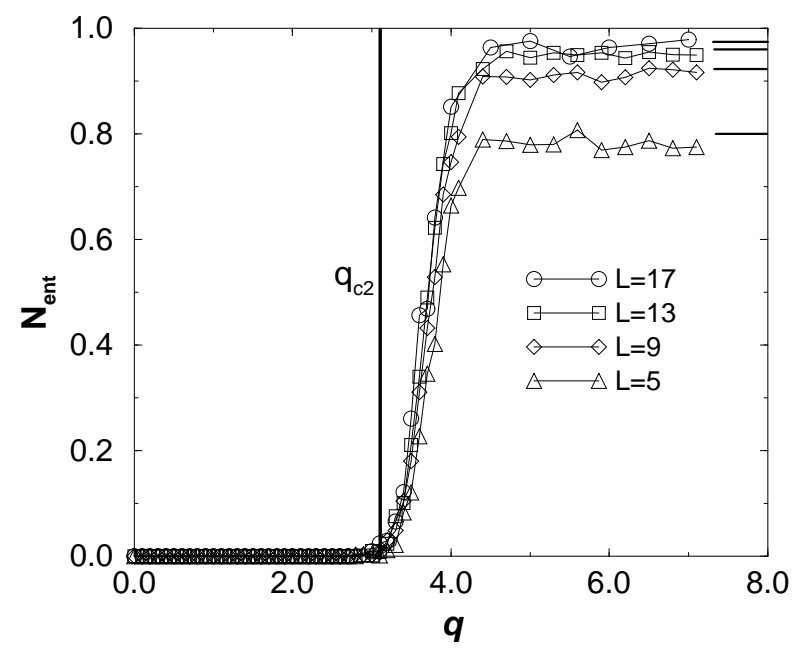

FIG. 6. Number of entangled lines $N_{\text {ent }}$ normalized by the total number of lines. The horrizontal lines indicate the analytically expected saturation values for random permutations of $N$ lines.

(1995).

[6] M. Lässig, J. Phys. C 10 (1998) 9905.

[7] M. Kardar, G. Parisi, and Y.-C. Zhang, Phys. Rev. Lett. 56, 889 (1986).

[8] L. Balents and M. Kardar Phys. Rev. B 49, 13030 (1994); T. Hwa and T. Nattermann, Phys. Rev. B 51, 455 (1995).

[9] G. Schröder et al., to be published.

[10] J.-Ph. Bouchaud and A. Georges, Phys. Rev. Lett. 68, 3908 (1992).

[11] T. Emig and T. Nattermann Phys. Rev. Lett. 81, 1469 (1998); Eur. Phys. J. B 8 525, (1999).

[12] P. Le Doussal and T. Giamarchi, Phys. Rev. Lett. 74 (1995) 606; J. Kierfeld, J. Physique 15 (1995) 379; J. P. Bouchaud, M. Mézard, and J. Yedidia, Phys. Rev. Lett. 67 (1991) 3840; T. Giarmachi and P. Le Doussal, Phys. Rev. Lett. 71 (1994) 1530.

[13] G. G. Batrouni and T. Hwa, Phys. Rev. Lett. 72 (1994) 4133; H. Rieger, Phys. Rev. Lett. 74 (1995) 4964. E. Marinari, R. Monasson, and J. J. Ruiz-Lorenzo, J. Phys. A 28 (1995) 3975; C. Zeng, A. A. Middleton, and Y. Shapir Phys. Rev. Lett. 77, (1996) 3204; H. Rieger and U. Blasum, Phys. Rev. B 55, R7394 (1997).

[14] T. Hwa, D. R. Nelson, and V. M. Vinokur. Phys. Rev. B 48, 1167 (1993)

[15] H. Rieger, Phys. Rev. Lett. 81, 4488 (1998).

[16] H. Rieger, Frustrated Systems: Ground State Properties via Combinatorial Optimization, Lecture Notes in Physics 501 (Springer Verlag Heidelberg, 1998); R. Ahuja, T. Magnanti, and J. Orlin, Network Flows, (Prentice Hall, New Jersey, 1993).

[17] M. Alava, P. Duxbury, C. Moukarzel, and H. Rieger, in Phase Transitions and Critical Phenomena, ed. C. Domb and J. L. Lebowitz (Academic Press, London, in press).

[18] T. Nattermann, I. Lyuksyutov, and M. Schwartz, Europhys. Lett. 16, 295 (1991). 